Article

\title{
Feature-Based Choice and Similarity Perception in Normal-Form Games: An Experimental Study
}

\section{Sibilla Di Guida ${ }^{1, *}$ and Giovanna Devetag ${ }^{2}$}

1 Solvay Brussels School of Economics and Management, European Center for Advanced Research in Economics and Statistics, Université Libre de Bruxelles, Avenue Roosevelt 50 CP 114, Bruxelles 1050, Belgium; E-Mail: sibilla.diguida@gmail.com

2 Luiss Guido Carli, Department of Business and Management, Viale Pola 12, Roma 00198, Italy; E-Mail: giovanna.devetag@gmail.com

* Author to whom correspondence should be addressed; E-Mail: sibilla.diguida@gmail.com; Tel.: +32-2-650-5950; Fax: +32-2-650-4475.

Received: 16 September 2013; in revised form: 5 December 2013 / Accepted: 12 December 2013 / Published: 18 December 2013

\begin{abstract}
In this paper, we claim that agents confronting with new interactive situations apply behavioral heuristics that drastically reduce the problem complexity either by neglecting the other players' incentives, or by restricting attention to subsets of "salient" outcomes. We postulate that these heuristics are sensitive to the manipulation of those features that can be modified without altering the (Nash) equilibrium structure of the game. We call these features "descriptive". We test experimentally the effect of these descriptive features on both choice behavior and cross-game similarity perception. Analysis of individual choices confirms our hypotheses, and suggests that non-equilibrium choices may derive from simplified mental models of the game structure, rather than from heterogeneous beliefs or limited iterative thinking. In addition, subjects tend to behave similarly in games sharing similar descriptive features, regardless of their strategic structure.
\end{abstract}

Keywords: cross-game similarity; categorization; focal point; behavioral heuristic; individual behavior; one-shot game; response time 


\section{Introduction}

Every day, people face several decisions; most of them have risible consequences and can be dealt with almost automatically, only a few are crucial. Many of these decisions, however, are new, i.e., they have never been encountered before. The situations people face in their daily routine are in fact often similar to other situations previously encountered, but rarely identical. Then, how do people react to a strategic situation they have never encountered before? When do people perceive two situations as being similar?

Behavioral game theory in the last decade has produced abundant experimental evidence in response to these questions, which in turn has informed empirically grounded modeling efforts. The enterprise has led to the development of new equilibrium concepts (e.g., Quantal Response Equilibrium [1], Cursed Equilibrium [2], to name a few) and of models that take on board individuals' limited reasoning abilities (i.e., the vast family of the level-k models [3-5]).

Behavioral models of game playing, although relaxing the most implausible assumptions of standard game theory, still rely on the notion of strategic thinking, i.e., they assume that players form beliefs about other players (in other words, players have a mental model of the other player's strategic behavior) and try to maximize their utility given these beliefs (an exception is given by level-0 players in level-k models, who are assumed to choose a strategy randomly).

Some of the available evidence, however, suggests a partly different picture of behavior in one-shot games, by pointing out that choice and belief might not be one the result of the other [6], by suggesting, more radically, that players may significantly underestimate their opponents' rationality [7], and finally, by hinting at the possibility that players may even act on the basis of a very simplified and/or incorrect model of the "true" strategic situation [8,9].

In this paper, we submit that players in several occasions may apply choice heuristics that imply a drastic simplification of the original decision problem, obtained either by ignoring the other players' motivations or by ignoring a subset of the game outcomes. These heuristics have the property of being "vulnerable" to the influence of features other than those pertaining to the game inner strategic structure: as a consequence, predictable changes in observed behavior can occur as a function of the presence vs. absence of these features.

More specifically, we argue that players in reasonably complex one-shot games, with no opportunity for learning and no feedback, at first look for "obvious" and "intuitive" solutions to the strategic problem they face: one such natural solution is picking a strategy which is both attractive and relatively safe, i.e., one with high payoff sum and low payoff variance. Alternatively, an equally "natural" solution is selecting the strategy supporting an outcome that is attractive for both players, which we call a focal point. The first heuristic is compatible with level-1 types in level-k models of strategic thinking [3-5] and may derive either from diffuse priors on the opponent's play or from a tendency to ignore opponents' moves entirely [7]. However, unlike in the models mentioned above, we assume that payoff variance (taken as an intuitive measure of the risk involved in choosing a strategy) plays an important role in determining level-1 behaviors. Although previous studies have investigated risk aversion in different economic settings [10-13], to the best of our knowledge, we are the first to test the role of payoff variance in influencing gaming behavior. The second heuristic is more strategic as it relies on Schelling salience [14-16] which has been identified in many previous experiments on 
matching games and which is well known for its effectiveness in promoting coordination. However, we assume that the effect of salience is much more widespread than commonly thought, even promoting the choice of focal points that are not equilibrium outcomes, as is the case in our games. Only in the absence of features suggesting attractive and intuitive solutions may players start to reason more strategically in a standard game-theoretic sense, and even find their way to equilibrium.

We also hypothesize that games sharing features such as the presence of a safe-and-attractive strategy and a focal point may trigger similar behaviors (at both the aggregate and individual levels), despite very different inner strategic structures; conversely, games which differ feature-wise but which present the same equilibrium properties may trigger very different behaviors. While similarity perception has been largely investigated in psychology [17,18], it was mostly neglected in economics (exceptions are [19-21]).

We claim that theories of cross-game similarity are crucial when modeling important phenomena such as cross-game transfer and generalization. It is widely acknowledged that the games we play in real life are at most similar to each other but never identical (unlike the typical "Groundhog day" lab situation) and, as many of our decision processes are case-based or analogy-based [22-24], it becomes essential to understand when players perceive two games as being similar. Surprisingly, there are very few studies investigating cross-game similarity perception. Among these, Grimm and Mengel [25] show that in a multiple games environment agents learn to extrapolate between games, categorize them according to their strategic structure, and consequently, play strategically equivalent games in the same way. Knez and Camerer [26] test transfer of precedent between a Prisoner's Dilemma and a Weak Link game, and introduce the distinction between surface (or descriptive) and structural similarity. In their design, transfer of precedent is triggered only in the presence of descriptively similar features between the two games (such as action labeling). Rankin et al. [27] test coordination behavior in perturbed environments by having subjects play a series of stag hunt games with randomly perturbed payoffs and action labels, and found that, when descriptive similarity is impeded, convergence to the payoff-dominant equilibrium is more frequent. Hence, understanding what features are relevant in eliciting similarity perception between games is crucial for modeling both repeated behaviors in ever-changing environments and phenomena of generalization from experience.

Showing that choice behavior responds to out-of-equilibrium (descriptive) features is obviously not a novel approach, and early examples date back to the famous studies of "framing" effects in individual decision making [28,29]. However, since our manipulations are "economic" in nature, implying exclusively changes in payoffs and, for one game only, changes in the position of the focal point in the matrix, they resemble more studies like Goeree and Holt [30].

Our feature manipulations influence behavior significantly and predictably, suggesting that players respond to features in the way hypothesized, and hence providing support to the hypothesis that players use the heuristics outlined. We also show that players respond similarly to games that are similar in terms of features, even when they belong to very different strategic types. Hence, a classification of games based on descriptive features (e.g., an outcome with symmetric, high payoffs, a strategy with high expected value and low variance, etc.) turns out to be more useful in predicting initial behavior than a categorization based on a game equilibrium structure.

Our findings are connected with previous studies in several ways: first, we provide evidence of behavior in one-shot normal form games which cannot be adequately explained by equilibrium 
concepts or by behavioral models which assume a distribution of game-invariant player types and thinking steps. In doing this, we point to the role of strategy variance as an important determinant of choice. Second, we extend the notion of a "focal point" well beyond equilibrium outcomes in symmetric games, showing that focality may be a much more general property of game outcomes, both symmetric and asymmetric.

More generally, we show that mild payoff changes induce relevant modifications in behavior, which can be parsimoniously explained by the use of decision heuristics that are based on a reduced model of the original problem, in line with suggestions from previous experiments [8]. We argue that these findings may constitute the basis for a theory of similarity that takes into account both structural and descriptive dimensions to describe players' cross-game similarity perceptions, the latter being more prominent than the former when initial or one-shot behavior is concerned.

Finally, our results add insights to the so-called "pre-game theory" [31], contributing to our understanding of strategic interaction situations as these are perceived and interpreted by the decision makers.

\section{The Games}

We define descriptive features as specific payoff manipulations that maintain the order relationships among payoffs and, consequently, do not affect the (Nash) equilibrium structure of the game. Specifically, we focus on two of these features: the introduction of a salient outcome (a "focal point”) and the manipulation of payoff variance in the strategy yielding the highest average payoff.

Our experimental design is aimed to test the following two hypotheses (to be expressed more formally after we define the games and relevant strategies):

Hypothesis 1 addresses the use of simple, intuitive, and feature-sensitive heuristics of choice. In particular, it tests whether: (a) a strategy with high average payoff and low payoff variance and a strategy leading to an out-of-equilibrium “focal point” are both more attractive than the equilibrium strategy, ceteris paribus; (b) when descriptive features are removed, the share of the corresponding strategy reduces significantly.

Hypothesis 2 addresses cross-game similarity perception. It tests whether: (a) a descriptive feature has a similar effect in strategically different games by influencing choice behavior in the same direction; and (b) keeping all other features fixed, the choice distributions in matrices which are strategically different but similar with respect to the features are closer - statistically - than the choice distributions of matrices which are strategically equivalent but differ with respect to the features.

In order to test our hypotheses, we used thirty $3 \times 3$ games in normal form belonging to five well-known game types. The payoff matrices used in the experiment are listed in Table 1 . The selected game categories are: a game with a strictly dominant strategy for the column player (henceforth, DomCol game), a game without pure strategy Nash Equilibria (noNE), a game with a single pure strategy Nash Equilibrium but not solvable through iterated elimination of dominated strategies 
Table 1. The games grouped by type, level of highest average payoff (HA) variance, and presence of Focal Point (FP). * indicates pure strategy Nash Equilibria. Frequency of each row is specified.

\begin{tabular}{|c|c|c|c|c|c|c|c|c|c|c|c|c|c|c|c|c|c|c|}
\hline \multirow{10}{*}{$\begin{array}{l}\overline{0} \\
\text { J } \\
\text { Oे }\end{array}$} & \multirow{5}{*}{ 全 } & \multicolumn{6}{|c|}{ HA low var } & \multicolumn{6}{|c|}{ HA middle var } & \multicolumn{5}{|c|}{ HA high var } \\
\hline & & & $\mathrm{C} 1$ & $\mathrm{C} 2$ & C3 & & & & $\mathrm{C} 1$ & C2 & C3 & & & & $\mathrm{C} 1$ & $\mathrm{C} 2$ & C3 & \\
\hline & & $\mathrm{R} 1$ & 35,20 & 35,25 & 35,30 & $45 \%$ & HA & $\mathrm{R} 1$ & 60,20 & 20,25 & 25,30 & $27 \%$ & HA & $\mathrm{R} 1$ & 80,20 & 10,25 & 15,30 & $23 \%$ \\
\hline & & R3 & 10,20 & 10,15 & $40,25 *$ & $17 \%$ & EQ & R3 & 10,20 & 10,15 & $40,25 *$ & $31 \%$ & EQ & R3 & 10,20 & 10,15 & $40,25 *$ & $34 \%$ \\
\hline & & & & FP & EQ/HA & & & & & FP & EQ/HA & & & & & FP & EQ/HA & \\
\hline & & & $\mathrm{C} 1$ & $\mathrm{C} 2$ & C3 & & & & $\mathrm{C} 1$ & C2 & C3 & & & & $\mathrm{C} 1$ & C2 & C3 & \\
\hline & & $\mathrm{R} 1$ & 35,20 & 35,25 & 35,30 & $80 \%$ & HA & $\mathrm{R} 1$ & 60,20 & 20,25 & 25,30 & $48 \%$ & HA & $\mathrm{R} 1$ & 80,20 & 10,25 & 15,30 & $33 \%$ \\
\hline & $\frac{\pi}{x}$ & $\mathrm{R} 2$ & 5,55 & 50,25 & 5,85 & $2 \%$ & FP & $\mathrm{R} 2$ & 5,55 & 50,25 & 5,85 & $7 \%$ & FP & $\mathrm{R} 2$ & 5,55 & 50,25 & 5,85 & $5 \%$ \\
\hline & & R3 & 10,20 & 10,15 & $40,25 *$ & $18 \%$ & EQ & R3 & 10,20 & 10,15 & $40,25 *$ & $45 \%$ & EQ & R3 & 10,20 & 10,15 & $40,25 *$ & $62 \%$ \\
\hline & & & & XFP & EQ/HA & & & & & XFP & EQ/HA & & & & & XFP & EQ/HA & \\
\hline \multirow{9}{*}{$\begin{array}{l}\text { 至 } \\
\stackrel{\varrho}{\varrho}\end{array}$} & & & $\mathrm{C} 1$ & $\mathrm{C} 2$ & C3 & & & & $\mathrm{C} 1$ & $\mathrm{C} 2$ & C3 & & & & $\mathrm{C} 1$ & $\mathrm{C} 2$ & C3 & \\
\hline & & R1 & 35,15 & 35,20 & 35,30 & $52 \%$ & HA & R1 & 55,15 & 25,20 & 25,30 & $37 \%$ & HA & R1 & 75,15 & 15,20 & 15,30 & $20 \%$ \\
\hline & & R3 & 15,35 & 5,25 & 40,20 & $16 \%$ & QES & R3 & 15,35 & 5,25 & 40,20 & $13 \%$ & QES & R3 & 15,35 & 5,25 & 40,20 & $22 \%$ \\
\hline & & & & $\mathrm{FP}$ & QES/HA & & & & & FP & QES/HA & & & & & FP & QES/HA & \\
\hline & & & $\mathrm{C} 1$ & C2 & C3 & & & & $\mathrm{C} 1$ & $\mathrm{C} 2$ & C3 & & & & $\mathrm{C} 1$ & $\mathrm{C} 2$ & C3 & \\
\hline & & $\mathrm{R} 1$ & 35,15 & 35,20 & 35,30 & $73 \%$ & HA & R1 & 55,15 & 25,20 & 25,30 & $53 \%$ & HA & $\mathrm{R} 1$ & 75,15 & 15,20 & 15,30 & $53 \%$ \\
\hline & $\sqrt{x}$ & $\mathrm{R} 2$ & 5,45 & 50,25 & 10,80 & $7 \%$ & XFP & $\mathrm{R} 2$ & 5,45 & 50,25 & 10,80 & $7 \%$ & XFP & $\mathrm{R} 2$ & 5,45 & 50,25 & 10,80 & $0 \%$ \\
\hline & & R3 & 15,35 & 5,25 & 40,20 & $20 \%$ & QES & R3 & 15,35 & 5,25 & 40,20 & $40 \%$ & QES & R3 & 15,35 & 5,25 & 40,20 & $47 \%$ \\
\hline & & & & XFP & QES/HA & & & & & XFP & QES/HA & & & & & XFP & QES/HA & \\
\hline \multirow{8}{*}{ 艺 } & & & C1 & $\mathrm{C} 2$ & C3 & & & & C1 & $\mathrm{C} 2$ & C3 & & & & $\mathrm{C} 1$ & C2 & C3 & \\
\hline & & $\mathrm{R} 1$ & 35,10 & 35,15 & 35,10 & $43 \%$ & HA & $\mathrm{R} 1$ & 55,10 & 25,15 & 25,10 & $28 \%$ & HA & $\mathrm{R} 1$ & 70,10 & 20,15 & 15,10 & $20 \%$ \\
\hline & 虹 & $\mathrm{R} 2$ & 10,50 & 70,70 & 5,75 & $47 \%$ & FP & $\mathrm{R} 2$ & 10,50 & 70,70 & 5,75 & $45 \%$ & FP & $\mathrm{R} 2$ & 10,50 & 70,70 & 5,75 & $43 \%$ \\
\hline & & R3 & 5,10 & 10,5 & $40,15 *$ & $10 \%$ & EQ & R3 & 5,10 & 10,5 & $40,15 *$ & $27 \%$ & EQ & R3 & 5,10 & 10,5 & $40,15 *$ & $37 \%$ \\
\hline & & & & FP & EQ/HA & & & & & $\mathrm{FP}$ & EQ/HA & & & & & FP & EQ/HA & \\
\hline & $\sqrt{x}$ & $\mathrm{R} 2$ & 10,50 & 50,25 & 5,75 & $13 \%$ & XFP & $\mathrm{R} 2$ & 10,50 & 50,25 & 5,75 & $3 \%$ & XFP & $\mathrm{R} 2$ & 10,50 & 50,25 & 5,75 & $12 \%$ \\
\hline & & R3 & 5,10 & 10,5 & $40,15 *$ & $12 \%$ & EQ & R3 & 5,10 & 10,5 & $40,15 *$ & $27 \%$ & EQ & R3 & 5,10 & 10,5 & $40,15 *$ & $41 \%$ \\
\hline & & & & XFP & EQ/HA & & & & & XFP & EQ/HA & & & & & XFP & EQ/HA & \\
\hline \multirow{10}{*}{ 全 } & & & $\mathrm{C} 1$ & C2 & C3 & & & & $\mathrm{C} 1$ & C2 & C3 & & & & $\mathrm{C} 1$ & C2 & C3 & \\
\hline & & $\mathrm{R} 1$ & 35,10 & 35,5 & $35,35 *$ & $87 \%$ & EQ/HA & $\mathrm{R} 1$ & 25,10 & 60,5 & $20,20 *$ & $80 \%$ & EQ/HA & $\mathrm{R} 1$ & 15,10 & 80,5 & $10,10 *$ & $80 \%$ \\
\hline & 公 & $\mathrm{R} 2$ & 10,35 & $35,35 *$ & 5,35 & $10 \%$ & $\mathrm{FP}$ & $\mathrm{R} 2$ & 10,35 & 35,35 & 5,60 & $17 \%$ & $\mathrm{FP}$ & $\mathrm{R} 2$ & 10,35 & 35,35 & 5,80 & $10 \%$ \\
\hline & & R3 & 15,15 & 35,10 & 10,35 & $3 \%$ & DOM & R3 & 15,15 & 35,10 & 10,25 & $3 \%$ & DOM & R3 & $15,15 *$ & 35,10 & $10,15 *$ & $10 \%$ \\
\hline & & & & FP & EQ/HA & & & & & FP & EQ/HA & & & & & FP & EQ/HA & \\
\hline & & & $\mathrm{C} 1$ & $\mathrm{C} 2$ & C3 & & & & $\mathrm{C} 1$ & C2 & C3 & & & & C1 & C2 & C3 & \\
\hline & & $\mathrm{R} 1$ & 35,10 & 35,5 & $35,35 *$ & $92 \%$ & $\mathrm{EQ} / \mathrm{HA}$ & $\mathrm{R} 1$ & 25,10 & 60,5 & $20,20 *$ & $87 \%$ & EQ/HA & $\mathrm{R} 1$ & 15,10 & 80,5 & $10,10 *$ & $68 \%$ \\
\hline & $\sqrt{x}$ & $\mathrm{R} 2$ & 10,35 & 35,25 & 5,35 & $5 \%$ & XFP & $\mathrm{R} 2$ & 10,35 & 35,25 & 5,60 & $5 \%$ & XFP & $\mathrm{R} 2$ & 10,35 & 35,25 & 5,80 & $10 \%$ \\
\hline & & R3 & 15,15 & 35,10 & 10,35 & $3 \%$ & DOM & R3 & 15,15 & 35,10 & 10,25 & $8 \%$ & DOM & R3 & $15,15 *$ & 35,10 & $10,15 *$ & $22 \%$ \\
\hline & & & & XFP & EQ/HA & & & & & XFP & EQ/HA & & & & & XFP & EQ/HA & \\
\hline \multirow{10}{*}{3} & \multirow{5}{*}{ 이 } & & $\mathrm{C} 1$ & $\mathrm{C} 2$ & C3 & & & & $\mathrm{C} 1$ & C2 & C3 & & & & C1 & $\mathrm{C} 2$ & C3 & \\
\hline & & $\mathrm{R} 1$ & $60,60 *$ & 35,45 & 5,35 & $57 \%$ & FP & $\mathrm{R} 1$ & $60,60 *$ & 35,45 & 5,35 & $58 \%$ & $\mathrm{FP}$ & $\mathrm{R} 1$ & $60,60 *$ & 35,45 & 5,35 & $10 \%$ \\
\hline & & $\mathrm{R} 2$ & 45,35 & $45,45 *$ & 35,35 & $42 \%$ & HA & $\mathrm{R} 2$ & 50,35 & $50,50 *$ & 20,35 & $33 \%$ & HA & $\mathrm{R} 2$ & 60,35 & $60,60 *$ & 5,35 & $72 \%$ \\
\hline & & R3 & 35,5 & 35,35 & $35,35 *$ & $1 \%$ & COS & R3 & 35,5 & 35,35 & $35,35 *$ & $9 \%$ & COS & R3 & 35,5 & 35,35 & $35,35 *$ & $18 \%$ \\
\hline & & & $\mathrm{FP}$ & HA & COS & & & & $\mathrm{FP}$ & HA & COS & & & & $\mathrm{FP}$ & HA & COS & \\
\hline & \multirow{5}{*}{$\frac{0}{x}$} & & $\mathrm{C} 1$ & $\mathrm{C} 2$ & C3 & & & & $\mathrm{C} 1$ & $\mathrm{C} 2$ & C3 & & & & $\mathrm{C} 1$ & $\mathrm{C} 2$ & C3 & \\
\hline & & $\mathrm{R} 1$ & 35,35 & $45,45 *$ & 45,35 & $48 \%$ & HA & $\mathrm{R} 2$ & 20,35 & $50,50 *$ & 50,35 & $38 \%$ & HA & $\mathrm{R} 2$ & 5,35 & $60,60 *$ & 60,35 & $65 \%$ \\
\hline & & $\mathrm{R} 2$ & 5,35 & 35,45 & $60,60 *$ & $48 \%$ & XFP & $\mathrm{R} 2$ & 5,35 & 35,45 & $60,60 *$ & $50 \%$ & XFP & $\mathrm{R} 2$ & 5,35 & 35,45 & $60,60 *$ & $12 \%$ \\
\hline & & R3 & $35,35 *$ & 35,35 & 35,5 & $4 \%$ & COS & R3 & $35,35 *$ & 35,35 & 35,5 & $12 \%$ & cos & R3 & $35,35 *$ & 35,35 & 35,5 & $23 \%$ \\
\hline & & & COS & HA & XFP & & & & COS & HA & XFP & & & & COS & HA & XFP & \\
\hline
\end{tabular}


(UniqNE), a $3 \times 3$ game with weakly dominant strategies similar to a Prisoners’ Dilemma (PD) ${ }^{1}$, and a Weak Link coordination game (WL).

Our main goal was to examine how the presence or absence of focal points affected subjects' perception of cross-game similarity and strategic behavior, as well as the effect of increasing the variance of the strategy with the highest average payoff (henceforth HA; three levels of variance were introduced: low, medium, high). For this purpose, and in order to identify both their separate and joint effects, we created a matrix for every possible combination of features: therefore, six matrices were created for each basic game. In few cases, in the differing versions of each game, new Nash equilibria emerged together with the original ones, which always remained.

For each game, we identified the strategy with the highest average payoff (HA), the equilibrium strategy (EQ, whenever a pure strategy Nash Equilibrium is present), and a strategy leading to a Focal Point (FP). We define as "focal" any outcome which yields identical payoffs to the players, is Pareto-efficient, but most of the times is not a Nash Equilibrium. Symmetry has already been shown as a crucial characteristic in Crawford et al. [32]. As we assume that players do not initially reason strategically in a game-theoretical sense, we consider more relevant for the attractiveness of an outcome to be Pareto-efficient rather than an equilibrium; moreover, it is important to disentangle the effects produced by purely descriptive features from those that might be produced by strategic reasoning. Using non-equilibrium focal points and focusing on focal points in games other than coordination games, we differentiate from most of the literature that mainly studies equilibrium focal points [15,32,33], or the effect of focal points in coordination games [15,32,34]. Motivated by the results of previous experiments [35], we located the focal point at the center of the matrix, except in the WL, where (due to the presence of three symmetric cells with increasing magnitude) the symmetric cells were positioned on the main diagonal in order of decreasing payoff magnitude.

To facilitate the exposition, we called each matrix by the acronym identifying the game type, and by two abbreviations identifying its features: FP means a matrix with a focal point, XFP a matrix without focal point, and low, middle and, high the three levels of variance of the strategy with the highest payoff sum. $^{2}$

All versions of each game were created, changing the cells' content as little as possible, and always preserving the game equilibrium structure. In a few cases, these changes added new Nash Equilibria in mixed strategies. In extreme cases, two matrices differed by a single cell. To avoid spurious effects due to the position of the strategy in the matrix, we always kept the position of every strategy fixed in the different versions of the same game, the only exception being the Weak Link game. Except in one matrix (WL_FP_low), the average payoff of the HA strategy was kept unchanged in the different

1 Our PD is not really a Prisoner's Dilemma, since it is a $3 \times 3$ game and does not contain strictly dominant strategies. However, we decided to name it PD for brevity, simplicity, and because the game nonetheless incorporates the basic tension between a cooperative strategy and a (weakly dominant) defecting strategy typical of a PD.

2 In addition, COS is a strategy yielding a constant payoff (present only in the Weak Link game) and DOM is a weakly dominated strategy (present only in the modified Prisoner’s Dilemma). QES is a quasi-equilibrium strategy since, according to Rubinstein [19] and Leland [20,21], C3 might be considered a dominant strategy for the column player (it dominates the other columns in Row 1 and Row 2, and it is only slightly smaller in Row 3). 
versions of the same game, and only the payoff distribution was modified so as to change the value of payoff variance.

We removed focal points in three different ways: first, as in games DomCol, noNE, and UniqNE, by breaking the symmetry of payoffs and reducing their magnitude, but maintaining the cell Pareto-efficient and its central position. Second, as in the Weak Link, by simply shifting strategy positions so as to place all the cells with symmetric payoffs outside the main diagonal. Third, as in the PD, by breaking the symmetry by only reducing the payoff of the column player. Since both payoffs were already relatively small, the payoff decrease in this case is slight.

In order to measure the impact of every feature, we kept our three strategies of interest separate whenever possible. For example, in the DomCol game, Row 1 identifies the HA strategy, Row 2 the FP strategy, and Row 3 the EQ strategy. This separation was not possible to obtain in the case of the Prisoner's Dilemma, where EQ and HA coincide by definition: in this case a single row is therefore simultaneously the EQ and the HA strategy. In addition, since keeping the three features of interest separate for both players was impossible, we chose to focus our analysis on the behavior of the row player only. Therefore, unless otherwise specified, all descriptions of strategies and matrices deal with the row player's perspective.

Finally, as we are interested in initial behavior only, we implemented a random rematching scheme with no feedback, to avoid learning and "repeated game effects" as much as possible.

\section{Experimental Design and Behavioral Predictions}

\subsection{Experimental Design and Implementation}

The experiment was conducted at the Computable and Experimental Economics Lab (CEEL) of the University of Trento, in five different sessions, each having 16 subjects. In each session, 12 subjects were randomly assigned the role of row player and four the role of column player, for a total of 60 observations for row players and 20 for column players. Roles were fixed throughout the experiment. Subjects made their choices as row or column players in the thirty matrices, and were re-matched randomly at every round with a player of the opposite role. All games were presented from the viewpoint of the row player. No feedback regarding opponents' choice or the obtained payoff was given until the end of the experiment.

On entering the lab, subjects were assigned randomly to a pc cubicle and to the role of row or column player. They were given a paper copy of the instructions ${ }^{3}$, which was also read aloud by the experimenter. Control questions were administered before starting the experiment, to ensure that the rules of the experiment had been understood. Particular care was taken to make sure that subjects understood how to read a payoff matrix. In the case of incorrect answers, instructions were repeated.

The experiment was computerized using a Z-Tree based software [36], specifically developed for the purpose. The matrices were presented one at a time, in random order, which differed across subjects. In each round, subjects had to select their preferred strategy by typing the corresponding row

3 See Figure 1 and the on-line appendix for a translated copy of the experimental instructions and of the questionnaire. 
number. At each decision stage, a message appeared after 30 seconds inviting subjects to make their choices. $^{4}$

The final payment was determined by the outcomes of five matrices picked at random. Subjects were informed about the minimum and maximum gain they could obtain, while the exchange rate was announced at the end. After the last matrix had been displayed, one subject selected randomly was asked to verify that tags in a jar each reported the numeric code of one of the matrices played. Subsequently, another randomly selected subject was asked to extract five tags from the jar to determine which matrices would be used for payments. Then all subjects were paid according to their own choices and their assigned partners' choices in the five selected matrices. ${ }^{5}$

Figure 1. Example of the software interface as it was presented to subjects in the instructions.

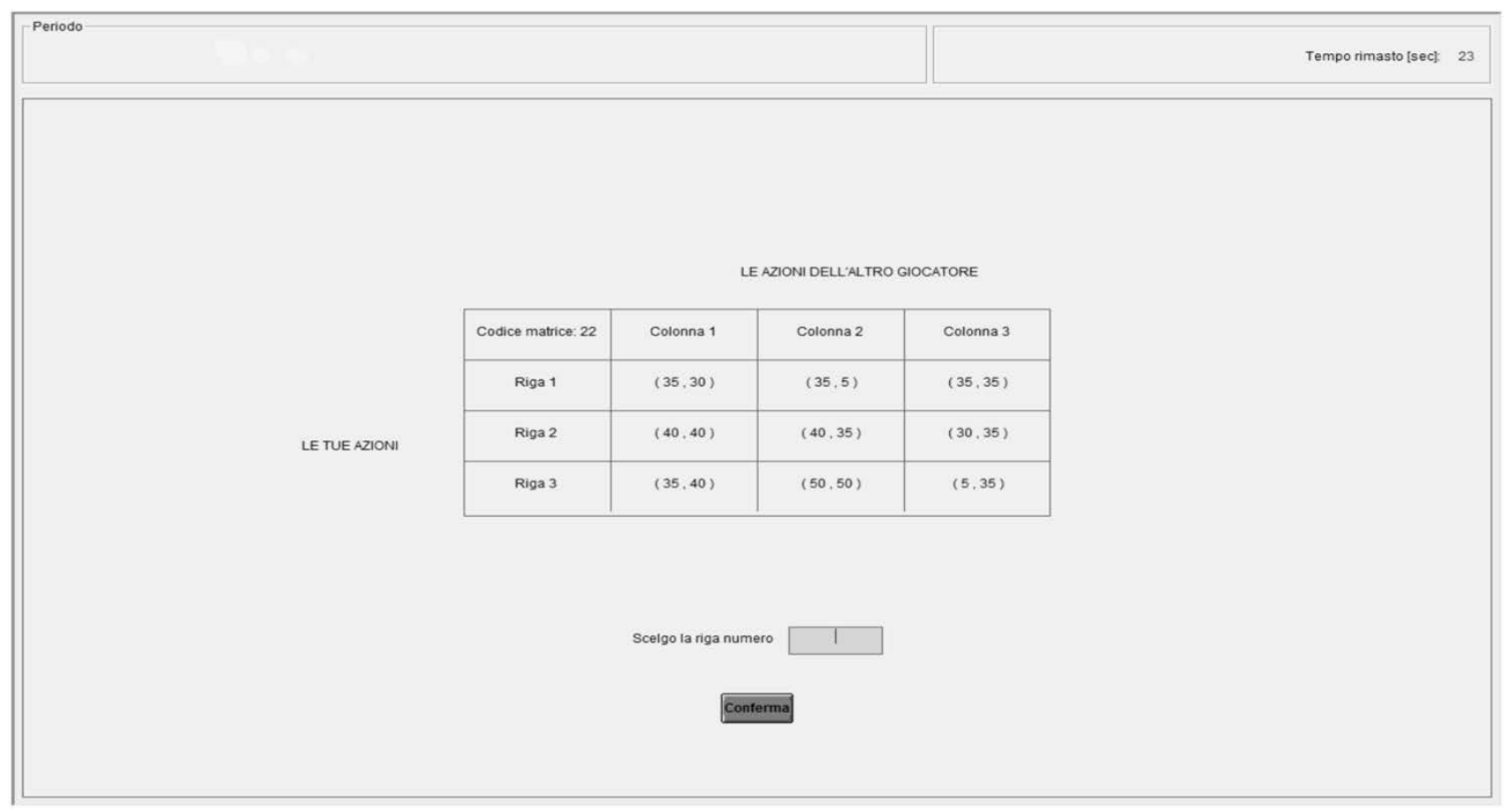

Before leaving the lab, subjects also took some personality tests and were administered the Holt and Laury lottery choices [12] with real payments. Hence, players' final payments were the sum of their earnings from the five matrices selected and their winnings from the lottery. Sessions did not last more than 90 min and average earnings equaled 14 Euros. Minimum earnings were equal to 10 Euros and maximum earnings were equal to 17.50 Euros. Average expected payment was calibrated according to the CEEL Lab guidelines.

\subsection{Behavioral Predictions}

4 We replicated the experiment without any recommendation concerning timing: all our findings on the behavior of the row players were replicated in the new experiment.

5 For each round and for each participant, the experimental software picked randomly a partner form the pool of players playing in the opposite role. Therefore if Row Player A was matched with Column Player 1, the opposite was not necessarily true, as the random matching was repeated for Column Player 1. 
Our choice of features has the goal of revealing the use of decision heuristics leading to intuitive solutions. We aim to extend our implications beyond choice, to cross-game similarity and transfer. We formulate specific hypotheses on the types of heuristics players may use in our games, as well as in other games that present the same or similar features: a heuristic that prescribes to choose the strategy with the best risk-return profile, and one that suggests picking the strategy leading to an attractive and fair outcome for both players.

To sum up, our experimental design is meant to test the following research hypothesis:

Hypothesis 1 ("Feature-Sensitive Strategic Behavior"): (a) when the variance of HA is low, strategies FP and HA capture the majority of choices in games with a focal point, and strategy HA captures the majority of choices in games without a focal point; (b) when the variance of HA increases, its share decreases, ceteris paribus; and (c) the share of the FP strategy in matrices with focal point is higher than the share of the corresponding strategy in matrices without focal point (XFP strategy).

Hypothesis 2 ("Feature-Based Similarity Perception"): (a) a "descriptive feature" has a similar effect in strategically different games by influencing choice behavior in the same direction; and (b) keeping all other features fixed, the choice distributions in matrices which are strategically different but similar with respect to the features are closer-statistically-than the choice distributions of matrices which are strategically equivalent but differ with respect to the features.

\section{Results and Discussion}

\subsection{Analysis of Aggregate Choices}

Table 1 reports the choice frequency for each row. Rows' frequencies are markedly different in the six versions of each game type, suggesting that feature manipulation influences behavior to a great extent. Furthermore, some patterns are clear-cut: specifically, the difference in observed frequencies between the same game with and without focal point is evident in most cases, as are the low shares of the EQ strategy (except for the Prisoner's Dilemma) and the effect of increasing the variance of HA. In particular, for each game, differences in the choice distributions of matrices with focal point and low variance (FP_low) and without focal point and high variance (XFP_high) - the two extreme casesare highly significant in all games, according to a chi-square test. ${ }^{6}$

\section{Hypothesis 1}

As hypothesized (Hypothesis 1a), when both features are strong (as in games with focal point and low variance) the corresponding strategies capture the large majority of players' choices (strategies HA low and FP are chosen at least $83 \%$ of the times). When the focal point is eliminated, HA increases its attractive power, capturing almost the same shares as in the previous case (HA low in XFP matrices is chosen at least $73 \%$ of the times). The only case which apparently contradicts our hypothesis is WL_XFP_low, in which $48 \%$ of subjects choose HA and another 48\% XFP. However, as previously specified, in the Weak Link the focal point was removed by simply moving the focal cell outside the main diagonal, with no changes in payoffs in order to preserve the game equilibrium structure. This

6 In the analysis that follows we define a difference "highly significant” when the corresponding p-value is less than 0.01 , and "significant" when it is less than 0.05 . 
evidence suggests that simply moving a focal point does not reduce its attractiveness: therefore the frequencies must be interpreted as $96 \%$ of players choosing strategies HA and FP, still in line with our hypothesis.

Table 2 reports data on the share of $\mathrm{HA}$ as a function of variance for the first four games (we discuss the Weak Link case separately). The share of HA decreases monotonically when the variance of HA increases from low, to medium, to high, supporting hypothesis $1 \mathrm{~b}$. We tested differences between matrices with HA low variance and those with HA high variance. For all matrices but PD_FP, the differences were significant. The case of the PD is particularly intriguing, since HA corresponds to EQ by construction, and is weakly dominant. Nevertheless, increasing the strategy variance without affecting its dominance induces a shift in behavior. On average, the frequency of HA passes from $68 \%$ (low variance case) to $43 \%$ (high variance case).

Table 2. Frequencies of HA choices and p-values obtained by comparing frequencies of strategies with low and high variance.

\begin{tabular}{lccccc}
\hline & HA low variance & HA middle variance & HA high variance & Chi-square test & $\begin{array}{c}\text { Binomial test } \\
\text { one-tailed }\end{array}$ \\
\hline DomCol FP & $45 \%$ & $27 \%$ & $23 \%$ & 0.02 & 0.01 \\
DomCol XFP & $80 \%$ & $48 \%$ & $43 \%$ & 0.00 & 0.00 \\
NoNE FP & $52 \%$ & $37 \%$ & $20 \%$ & 0.01 & 0.00 \\
NoNE XFP & $73 \%$ & $53 \%$ & $53 \%$ & 0.00 & 0.02 \\
UniqNE FP & $43 \%$ & $28 \%$ & $20 \%$ & 0.00 & 0.00 \\
UniqNE XFP & $75 \%$ & $68 \%$ & $47 \%$ & 0.00 & 0.00 \\
PD FP & $87 \%$ & $80 \%$ & $80 \%$ & 0.34 & 0.23 \\
PD XFP & $92 \%$ & $87 \%$ & $68 \%$ & 0.00 & 0.00 \\
\hline
\end{tabular}

A different approach must be used for the Weak Link game. Here, the effect of variance cannot be observed directly, but it must be inferred from the share of strategy COS (the strategy giving a constant payoff). Due to equilibrium constraints, while in HA low variance and HA middle variance strategies HA and FP are distinct, in HA high variance two focal points appear: one in the former FP strategy and another in HA. Therefore, instead of testing whether increasing the variance of HA reduces its share, we verify whether it increases the share of COS (the only strategy without focal points). In WL_FP, the frequency of COS passes from $2 \%$ in the low variance matrix, to $8 \%$ in the medium variance matrix, to $18 \%$ in the high variance matrix, whereas in WL_XFP, the frequency rises from $3 \%$, to $12 \%$, to $23 \%$. In both cases, chi-square and binomial tests showed that the differences between low and high variance matrices were highly significant. We conclude that, in WL also, our hypothesis (1b) is confirmed.

Concerning the role of the focal point in our matrixes (hypothesis 1c), it is noteworthy that the share of FP is always higher (and equal in only one case) than the share of XFP. The frequencies of FP, XFP, and the corresponding p-values are listed in Table 3. In the first three game categories-DomCol, noNE and UniqNE - the average difference in share between FP and XFP is 38\%. In the case of PD and WL, it falls to $6.5 \%$, and is $25.4 \%$ overall. We made pairwise comparisons of the choice distributions with a chi-square test. The hypothesis was confirmed for games DomCol, noNE and UniqNE, and the difference were highly significant in all nine comparisons. In PD also, the frequencies of XFP were always smaller than or equal to the corresponding frequencies of $\mathrm{FP}$, but the 
differences were not significant. There are two reasons for this difference: first and most importantly, the focal point in game $\mathrm{PD}$ is weaker than in remaining games, due to a lower relative payoff magnitude: consequently, the related strategy is chosen by fewer subjects than in any other game. Second, in the PD, the focal point is eliminated only by breaking the symmetry, with a minimal change in payoff magnitude for the column player and no changes in the payoff of the row player. In WL also, FP frequencies are higher than those of XFP, although the differences are not statistically significant. As previously said, in the Weak Link, XFP is obtained by simply shifting the cell position without altering its content. This change apparently does not significantly affect the cell focality. ${ }^{7}$

Table 3. Frequencies of FP and XFP choices for row players, and corresponding $p$-values.

\begin{tabular}{lcccc}
\hline & FP & XFP & chi-square test & Binomial test one-tailed \\
\hline DomCol HA low & $38 \%$ & $2 \%$ & 0.00 & 0.00 \\
DomCol HA middle & $42 \%$ & $7 \%$ & 0.00 & 0.00 \\
DomCol HA high & $43 \%$ & $5 \%$ & 0.00 & 0.00 \\
noNE HA low & $32 \%$ & $7 \%$ & 0.00 & 0.00 \\
noNE HA middle & $50 \%$ & $7 \%$ & 0.00 & 0.00 \\
noNE HA high & $58 \%$ & $0 \%$ & 0.00 & 0.00 \\
UniqNE HA low & $47 \%$ & $13 \%$ & 0.00 & 0.00 \\
UniqNE HA middle & $45 \%$ & $3 \%$ & 0.00 & 0.00 \\
UniqNE HA high & $43 \%$ & $12 \%$ & 0.00 & 0.00 \\
PD HA low & $10 \%$ & $5 \%$ & 0.58 & 0.24 \\
PD HA middle & $17 \%$ & $5 \%$ & 0.07 & 0.04 \\
PD HA high & $10 \%$ & $10 \%$ & 0.20 & 0.50 \\
WL HA low & $57 \%$ & $48 \%$ & 0.60 & 0.46 \\
WL HA middle & $58 \%$ & $50 \%$ & 0.62 & 0.46 \\
WL HA high & $82 \%$ & $77 \%$ & 0.73 & 0.65 \\
\hline
\end{tabular}

As regards the importance of the focal point, the behavior of the column players is particularly interesting. The DomCol game presents a strictly dominant strategy for the column player, whereas both noNE and UniqNE present a strategy yielding the highest payoff in 2 out of 3 cells and a slightly lower payoff in the third cell: hence, a large share of FP on the part of column players indicates that its importance is considerable, in view of the available alternatives. The frequencies of FP, XFP, and of the (quasi-)dominant strategies for column players are listed in Table 4. When the focal point is present, a share that goes from $25 \%$ to $60 \%$ of column players chooses FP, but less than $30 \%$ violates dominance when the focal point is absent. The choice of FP on the part of these players cannot therefore be attributed to error or confusion. Altogether, these results confirm our hypothesis 1c and show that, when the difference between FP and XFP outcomes is evident, the effect on subjects' choice behavior is significant. ${ }^{8}$

Hypothesis 2

\footnotetext{
We remind that the frequency "WL HA high" is obtained by summing the frequencies of FP and HA, since-for structural reasons - two identical focal points appear in that matrix, one for each of these strategies.

8 See the on-line appendix for an extensive analysis of focal points and their characteristics.
} 
Our aim in this study is to show that observed differences in strategies among games sharing the same equilibrium structure follow predictable patterns governed by the presence vs. absence of the descriptive features defined above, which reveal the use of simple decision heuristics on the part of our subjects.

Table 4. Frequencies of FP and XFP choices for column players, and corresponding $p$ values. In brackets, choice frequencies of EQ and QES strategies in the corresponding matrices.

\begin{tabular}{lccc}
\hline & FP (EQ) & XFP (EQ) & Binomial test one-tailed \\
\hline DomCol HA low & $30 \%(70 \%)$ & $5 \%(95 \%)$ & 0.05 \\
DomCol HA middle & $50 \%(50 \%)$ & $0 \%(100 \%)$ & $<0.001$ \\
DomCol HA high & $35 \%(65 \%)$ & $5 \%(95 \%)$ & 0.02 \\
noNE HA low & $25 \%(75 \%)$ & $0 \%(100 \%)$ & 0.03 \\
noNE HA middle & $45 \%(55 \%)$ & $0 \%(100 \%)$ & $<0.001$ \\
noNE HA high & $30 \%(70 \%)$ & $5 \%(90 \%)$ & 0.05 \\
UniqNE HA low & $60 \%(40 \%)$ & $15 \%(70 \%)$ & $<0.001$ \\
UniqNE HA middle & $45 \%(55 \%)$ & $30 \%(70 \%)$ & 0.26 \\
UniqNE HA high & $60 \%(40 \%)$ & $25 \%(70 \%)$ & 0.03 \\
\hline
\end{tabular}

As said beforehand, for all our game types, the difference in choice shares between the matrix with all features and that without features is always highly significant. A focal point (according to our definition) is one of these features. We have shown that, even when FP is a strictly dominated strategy, it can still attract a significant fraction of players' choices. Another feature that influences strategic behavior is the strategy giving the highest average payoff (HA) when it is perceived as a "safe" option (low variance). In this case too, HA determines similar effects in different games, and the importance of the "safety" attribute is revealed by the emergence of an inverse relationship between the share of players choosing HA and its variance level.

Altogether, our results show that the analyzed features affect behavior in the same direction, regardless of the game-theoretical properties of the strategic situation at hand, supporting hypothesis 2a. Therefore, it may be hypothesized that strategically different games are perceived as similar when they share some or all of these features.

In hypothesis $2 \mathrm{~b}$, we propose that games sharing the same features generate choice distributions that are so similar to be statistically indistinguishable (we omit the Prisoner's Dilemma and Weak Link because, comparison wise, their strategic structure is too different). Table 5 shows that, in most of the comparisons, frequency distributions in games sharing the same features do not appear to be significantly different. Hence, whereas frequencies differ significantly when the same game type is compared with and without features (as shown in hypothesis 2a), when the latter remain unaltered but the game structure changes, players' strategic behavior remains largely invariant, suggesting that the difference is not perceived as such in the aggregate. In further support to our hypothesis, it must be noted that the frequencies of DomCol, noNE, and UniqNE are all significantly different (according to a chi-square test) from one another only in the XFP high var case, when all features are removed and hence the underlying equilibrium structure may result more clearly visible. 


\subsection{Analysis of Individual Behavior}

In this section, we investigate the effect of features on individual behavior first by applying the model presented in Costa-Gomes et al. [37] to our data, then defining new types based on features, and finally comparing the performances of the two models.

Table 5. Comparison of games with the same key features and different strategic structures (p-values).

\begin{tabular}{cccc}
\hline & & \multicolumn{2}{c}{ Chi-square test } \\
\cline { 3 - 4 } & & noNE & UniqNE \\
\hline \multirow{2}{*}{ HA low var FP } & DomCol & 0.72 & 0.47 \\
& noNE & & 0.21 \\
\hline \multirow{2}{*}{ HA middle var FP } & DomCol & 0.05 & 0.83 \\
& noNE & & 0.18 \\
\hline \multirow{2}{*}{ HA high var FP } & DomCol & 0.23 & 0.88 \\
& noNE & & 0.16 \\
\hline \multirow{2}{*}{ HA low var XFP } & DomCol & 0.36 & 0.04 \\
& noNE & & 0.26 \\
\hline \multirow{2}{*}{ HA middle var XFP } & DomCol & 0.85 & 0.08 \\
& noNE & & 0.23 \\
\hline \multirow{2}{*}{ HA high var XFP } & DomCol & 0.03 & 0.07 \\
& noNE & & 0.02 \\
\hline
\end{tabular}

Costa-Gomes et al. [37] identify nine types of strategic behavior, which summarize a wide range of possible decision rules a player can apply in a game: Altruistic (an agent aiming at the cell that maximizes the sum of his own and his opponent's payoff), Pessimistic (an agent choosing the strategy with the highest minimum payoff), Naïve (an agent picking the strategy with the highest average payoff, under the assumption that the opponent's choices are equally likely), Optimistic (a player aiming at the highest payoff for herself), L2 (an agent who best responds to a Naïve opponent), D1 (an agent who is able to single out a dominated strategy and then assign equal probability to the remaining choices of her opponent), D2 (an agent performing two rounds of iterated elimination of dominated strategies), Equilibrium (an agent who selects equilibrium strategies), Sophisticated (an agent who best responds to the probability distribution of his opponent's decisions).

In their paper, the authors conduct a maximum likelihood error-rate analysis of agents' decisions. Their econometric model is a mixture model that assumes common prior distribution of types, and assigns to each type a probability of error (trembling hand). They define the model as follows:

$i=1, \ldots, \mathrm{N}$ indexes the agents, $k=1, \ldots, \mathrm{K}$ indexes the types, and $\mathrm{c}$ is the number of agents' possible decisions ( $c=3$ in all our matrices). Type-k agents are assumed to act coherently, but they might make an error with probability $\varepsilon_{\mathrm{k}} \in[0,1]$ (type k's error rate), in which case each of the c possible decisions has probability 1/c. Therefore, for a type-k agent the probability of a coherent choice is $1-(c-1) \varepsilon_{\mathrm{k}} / c$, whereas the probability of any single incoherent one is $\varepsilon_{\mathrm{k}} / c$. Errors are assumed to be independently and identically distributed across games and subjects. 


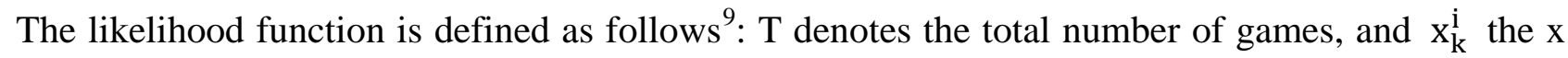
choices that subject $\mathrm{i}$ made coherently with type $k$, with $\mathrm{x}^{\mathrm{i}} \equiv\left(\mathrm{x}_{1}^{\mathrm{i}}, \ldots, \mathrm{x}_{\mathrm{K}}^{\mathrm{i}}\right)$, and $\mathrm{x} \equiv\left(\mathrm{x}^{1}, \ldots, \mathrm{x}^{\mathrm{N}}\right)$. Let $\mathrm{p} \equiv\left(\mathrm{p}_{1}, \ldots, \mathrm{p}_{\mathrm{K}}\right)$, with $\sum_{\mathrm{k}=1}^{\mathrm{K}} \mathrm{p}_{\mathrm{k}}=1$, denote subjects' common prior probabilities, and $\varepsilon \equiv\left(\varepsilon_{1}, \ldots, \varepsilon_{\mathrm{K}}\right)$ the types' error rates. Each type-k identifies one and one only decision in each game, whereas all other c -1 are non-type-k decisions. Therefore, the probability of observing a specific sample with $x_{k}^{i}$ type-k decisions when subject $\mathrm{i}$ is type $\mathrm{k}$ is:

$$
\mathrm{L}_{\mathrm{k}}^{\mathrm{i}}\left(\varepsilon_{\mathrm{k}} \mid \mathrm{x}_{\mathrm{k}}^{\mathrm{i}}\right)=\left[1-(\mathrm{c}-1) \varepsilon_{\mathrm{k}} / \mathrm{c}\right]^{\mathrm{x}_{\mathrm{k}}^{\mathrm{i}}}\left[\varepsilon_{\mathrm{k}} / \mathrm{c}\right]^{\mathrm{T}-\mathrm{x}_{\mathrm{k}}^{\mathrm{i}}}
$$

That leads to the following log-likelihood function for the whole sample:

$$
\ln L(p, \varepsilon \mid x)=\sum_{i=1}^{N} \ln \left[\sum_{k=1}^{K} p k\left[1-(c-1) \varepsilon_{k} / c\right]^{x_{k}^{i}}\left[\varepsilon_{k} / c\right]^{T-x_{k}^{i}}\right]
$$

With nine types, the model has seventeen parameters: eight independent type probabilities $\mathrm{p}_{\mathrm{k}}$, and nine type-dependent error rates $\varepsilon_{\mathrm{k}}$.

In order to apply the model to our data, we proceeded by classifying each choice according to the pre-defined set of strategic types defined in Costa-Gomes et al. [37]. Out of the thirty payoff matrices we used, seventeen had to be excluded as the same strategic type would have selected multiple strategies.

Of the nine types presented in Costa-Gomes et al., only five could be applied to our games. Types D1, D2, and L2 always coincided with Equilibrium, therefore we simply created the Equilibrium type, which includes them all. Pessimistic and Naïve coincided as well, and we labeled the corresponding type Pessimistic/Naïve. The five resulting strategic types are then: Altruistic (A), Pessimistic/Naïve $(\mathrm{P} / \mathrm{N})^{10}$, Optimistic $(\mathrm{O})^{11}$, Equilibrium (E), and Sophisticated (S).

To test whether agents are influenced in their choices by features, we identify four feature-based types: Naïve (already defined in [37]), Focal/Naïve (F/N, an agent who chooses the Focal Point whenever possible and switches to the strategy with the highest average payoff otherwise), Focal/Equilibrium (F/E, an agent who chooses the Focal Point whenever possible and switches to the equilibrium strategy otherwise), and Nä̈ve/Equilibrium (N/E, an agent who chooses the strategy with the highest average payoff when that strategy is riskless and switches to the equilibrium strategy otherwise). Table 6 reports the matrices of interest and each type's strategy.

We computed maximum likelihood estimates of the parameters, first for the Costa-Gomes et al.'s types, and then for our four feature-base types plus the Equilibrium one. Table 7 reports the estimations results.

9 The original likelihood function allowed for matrices to have different dimensions. As in our paper all matrices are $3 \times 3$, we report only the simplified version.

10 If more strategies were selected, then the one with the second highest minimum was chosen.

11 If more strategies were selected, we chose the one in a more relevant position (top-left cell). 
Table 6. Matrices used for the individual analysis, with each type's choice specified.

\begin{tabular}{|c|c|c|c|c|c|c|c|}
\hline \multicolumn{2}{|c|}{ DomCol_FP_low } & \multicolumn{2}{|c|}{ DomCol_FP_middle } & \multicolumn{2}{|c|}{ DomCol_FP_high } & \multicolumn{2}{|c|}{ DomCol_XFP_low } \\
\hline R1 & $\mathrm{P} / \mathrm{N}, \mathrm{S}, \mathrm{N} / \mathrm{E}$ & R1 & $\mathrm{P} / \mathrm{N}$ & R1 & $\mathrm{P} / \mathrm{N}, \mathrm{O}$ & R1 & $\mathrm{P} / \mathrm{N}, \mathrm{N} / \mathrm{E}, \mathrm{F} / \mathrm{N}$ \\
\hline $\mathbf{R} 2$ & $\mathrm{~A}, \mathrm{O}, \mathrm{F} / \mathrm{N}, \mathrm{F} / \mathrm{E}$ & $\mathbf{R 2}$ & $\mathrm{A}, \mathrm{O}, \mathrm{S}, \mathrm{F} / \mathrm{N}, \mathrm{F} / \mathrm{E}$ & $\mathbf{R 2}$ & $\mathrm{A}, \mathrm{S}, \mathrm{F} / \mathrm{N}, \mathrm{F} / \mathrm{E}$ & $\mathbf{R} 2$ & $\mathrm{~A}, \mathrm{O}$ \\
\hline R3 & E & R3 & E, N/E & R3 & E, N/E & R3 & $\mathrm{E}, \mathrm{S}, \mathrm{F} / \mathrm{E}$ \\
\hline \multicolumn{2}{|c|}{ DomCol_XFP_middle } & \multicolumn{2}{|c|}{ DomCol_XFP_high } & \multicolumn{2}{|c|}{ UniqNe_FP_low } & \multicolumn{2}{|c|}{ UniqNE_FP_middle } \\
\hline R1 & $\mathrm{P} / \mathrm{N}, \mathrm{O}, \mathrm{F} / \mathrm{N}$ & R1 & $\mathrm{P} / \mathrm{N}, \mathrm{A}, \mathrm{O}, \mathrm{F} / \mathrm{N}$ & R1 & $\mathrm{P} / \mathrm{N}, \mathrm{N} / \mathrm{E}$ & R1 & $\mathrm{P} / \mathrm{N}$ \\
\hline $\mathbf{R} 2$ & A & $\mathbf{R} 2$ & & $\mathbf{R} 2$ & $\mathrm{~A}, \mathrm{O}, \mathrm{S}, \mathrm{F} / \mathrm{N}, \mathrm{F} / \mathrm{E}$ & $\mathbf{R} 2$ & $\mathrm{~A}, \mathrm{O}, \mathrm{S}, \mathrm{F} / \mathrm{N}, \mathrm{F} / \mathrm{E}$ \\
\hline R3 & $\mathrm{E}, \mathrm{S}, \mathrm{N} / \mathrm{E}, \mathrm{F} / \mathrm{E}$ & R3 & E, S, N/E, F/E & R3 & & R3 & E, N/E \\
\hline \multicolumn{2}{|c|}{ UniqNE_FP_high } & \multicolumn{2}{|c|}{ UniqNE_XFP_low } & \multicolumn{2}{|c|}{ UniqNE_XFP_middle } & \multicolumn{2}{|c|}{ PD_FP_middle } \\
\hline R1 & $\mathrm{P} / \mathrm{N}, \mathrm{O}$ & $\mathbf{R} 1$ & $\mathrm{P} / \mathrm{N}, \mathrm{S}, \mathrm{N} / \mathrm{E}, \mathrm{F} / \mathrm{N}$ & $\mathbf{R 1}$ & $\mathrm{P} / \mathrm{N}, \mathrm{S}, \mathrm{F} / \mathrm{N}$ & R1 & $\mathrm{E}, \mathrm{P} / \mathrm{N}, \mathrm{O}, \mathrm{S}, \mathrm{N} / \mathrm{E}, \mathrm{F} / \mathrm{N}, \mathrm{F} / \mathrm{E}$ \\
\hline $\mathbf{R} 2$ & $\mathrm{~A}, \mathrm{~S}, \mathrm{~F} / \mathrm{N}, \mathrm{F} / \mathrm{E}$ & $\mathbf{R} 2$ & A, O & $\mathbf{R} 2$ & $\mathrm{~A}, \mathrm{O}$ & $\mathbf{R} 2$ & A \\
\hline R3 & E, N/E & R3 & $\mathrm{E}, \mathrm{F} / \mathrm{E}$ & R3 & E, N/E, F/E & R3 & \\
\hline \multicolumn{2}{|c|}{ PD_XFP_low } & & & & & & \\
\hline R1 & E, P/N, A, O, S, N/E, F/N, F/E & & & & & & \\
\hline $\mathbf{R} 2$ & & & & & & & \\
\hline R3 & & & & & & & \\
\hline
\end{tabular}

Table 7. Parameters estimated using Costa-Gomes et al. types and with feature-based types. Log-likelihood in brackets.

\begin{tabular}{c|cccccc}
\hline Costa-Gomes et al. & & Altruistic & Pessimistic/Naïve & Optimistic & Equilibrium & Sophisticated \\
Types & Estimated freqency & 0.046 & 0.286 & 0.000 & 0.142 & 0.524 \\
$(-712.19)$ & Error rates & 0.490 & 0.332 & - & 0.410 & 0.614 \\
\hline \multirow{2}{*}{ Feature-based types } & & Naïve & Focal/Naïve & Focal/Equilibrium & Naïve/Equilibrium & Equilibrium \\
$(-668.08)$ & Estimated freqency & 0.244 & 0.298 & 0.106 & 0.290 & 0.062 \\
& Error rates & 0.284 & 0.462 & 0.488 & 0.460 & 0.301 \\
\hline
\end{tabular}

To test which of the two models best fits the data, we calculated the second-order Akaike's information criterion $(\mathrm{AICc})^{12}$ [38], defined as:

$$
\operatorname{AIC}_{\mathrm{c}}=-2 \log (\mathcal{L}(\widehat{\theta}))+2 \mathrm{~K}(n / n-K-1)
$$

where $\mathcal{L}(\widehat{\theta})$ is the maximum log-likelihood, $\mathrm{K}$ the number of estimable parameters, and $\mathrm{n}$ the number of subjects (sample size).

AICc for the feature-base model is $\operatorname{AICc}(\mathrm{fb})=1360.16$, while for the Costa-Gomes et al. model is $\mathrm{AICc}(\mathrm{ccb})=1448.38$. As its AICc is smaller, the feature-based model is the one that best fits the data [38].

Furthermore, since $\Delta_{c c b}=\operatorname{AICc}(\mathrm{ccb})-\operatorname{AICc}(\mathrm{fb})>10$, data give no empirical support to the Costa-Gomes et al.'s model in comparison to the feature-based one [38]. ${ }^{13}$

Overall, our model captures subjects' behavior better, and supports the hypothesis of feature-based choice since $70 \%$ of subjects switch type according to the games features (F/N, F/E, and N/E). In

12 Given the number of parameters and the sample size, AICc is a better estimator than AIC.

13 According to [38], for $\Delta_{i}=A I C c_{i}-A I C c_{\min }$, the level of empirical support of Model $i$ compared to Model min is: substantial for $\Delta_{i}$ from 0 to 2; considerably less for $\Delta_{i}$ from 4 to 7; essentially none for $\Delta_{i}>10$. 
addition, the fact that almost $60 \%$ of the subjects behave as naïve players only in certain conditions (F/N and N/E) suggests that level-1 behavior might not be produced by lack of iterated reasoning capacities, but by a deliberate strategy.

Finally, in order to test whether different choices were more "strategic" than others, we calculated the correlation between an individual's strategic IQ (following the method proposed in Bhatt and Camerer [39]) and the number of HA/FP/EQ choices made in the game. We find that strategic IQ negatively correlates with the choice of HA, while it is positively correlated with the choice of a focal point and with equilibrium choices ${ }^{14}$. These results do not change if we consider spurious combinations such as $\mathrm{HA} / \mathrm{EQ}$, etc.

\subsection{Analysis of Response Times}

To gain further insight into subjects' choice processes, we analyze differences in response times. Some recent studies of gaming behavior employ response time as means to explore subjects' decision-making processes [40,41]. In a recent fMRI study on gaming behavior, Kuo et al. [42] found that subjects took a much longer time, on average, to choose a strategy in dominance-solvable games than in coordination games, and different areas of the brain were activated when players faced instances of the two classes of games. According to these findings, the authors suggested the existence of two different "strategizing" systems in the brain, one based on analytical reasoning and deliberation and the other on intuition and a "meeting of the minds".

Along the lines of Kuo et al. [42], we hypothesize that matrices with descriptive features are approached using heuristics and hence require a shorter response time than matrices without features, which are presumed to require analytical reasoning.

The individual response time for matrices with focal point is (highly) significantly shorter than that for matrices without focal point. ${ }^{15}$ Note that the significance of results holds, although some subjects did not select the focal point strategy in the matrices which contained it, and those who did not presumably employed the same type of analytical reasoning used for games without focal point.

We also observed an increase in response time when the variance of the HA strategy increases. Response time averages 17.71 in the low variance case, 20.98 in the middle variance case, and 23.66 in the high variance case. Pairwise differences of individual response time are highly significant. ${ }^{16}$ Finally, a positive significant correlation was found between individual response times and number of EQ choices. $^{17}$

14 Values of the Spearman correlation coefficients are: -0.503 for HA ( $p$-value $<0.01)$, 0.284 for FP ( $p$-value $<0.05$ ), and 0.466 for EQ ( $p$-value < 0.01). We also calculated correlation between choices in the games and Holt and Laury [12] risk aversion index. Risk aversion is positively correlated with equilibrium choices and with strategic IQ, whereas no correlation emerges between risk attitudes and choices of HA and FP. No correlation was also found between risk attitudes and variance levels of HA.

15 Paired $t$-test, two-tailed; same results are obtained by a non-parametric Wilcoxon signed rank test

16 Paired $t$-test, two-tailed; same results are obtained by a non-parametric Wilcoxon signed rank test.

17 Spearman's rho coeff $=0.273$, two-tailed. 
Summarizing, not only subjects switch from one type to another when features are removed (according to our model), but also increase the time required to take a decision. Altogether, these findings support the hypothesis of subjects using feature-based heuristics to approach the games, and switch to a more sophisticated analysis when the heuristics are not conclusive.

\section{Conclusions}

This paper shows that behavior in one-shot games may be explained by a set of very simple heuristics. These heuristics imply a drastic simplification of the situation at hand, and as such are affected by the manipulation of what we define as "descriptive features".

More specifically, we show that the attractive power of focal points extends to asymmetric games and non-equilibrium outcomes, and identify two attributes (payoff symmetry and payoff magnitude), which, when jointly present, are the two factors most frequently responsible for making an outcome focal. We also show that the presence of a strategy with high expected value and low variance (a "safe", attractive strategy) is a strong choice attractor. Together, the strategy yielding the focal point and the safe strategy explain most of players' choices.

The two heuristics investigated have intuitive appeal and seem to be "activated" by the presence of features. As a consequence, players should be likely to behave similarly in games that share one of more features, regardless of cross-game structural (i.e., equilibrium) similarity. This is what happens in our experiment: we show that subjects react in similar ways to different game types sharing the same features. Hence, in our experiment, with reasonably complex matrices presented randomly, the use of the same heuristics repeatedly may have been determined both by the salience of the features themselves and by the players’ referring to past experience with similar matrices.

\section{Acknowledgments}

We thank Christopher Gilbert, Paola Manzini, Marco Piovesan, Ondrej Rydval, Theodore L. Turocy, Enrico Zaninotto, participants at the IMEBE 2010 in Bilbao, and seminar participants in Trento for their useful comments and suggestions. We also thank Franz Dietrich, Ido Erev, Hykel Hosni, Ariel Rubinstein, Karl Schlag and other participants at the Workshop on Rationality, Heuristics and Motivation in Decision Making, Scuola Normale Superiore, Pisa, Italy, 12-14 November 2010. We thank the staff of the CEEL experimental laboratory for help in implementing experiments. We gratefully acknowledge financial support from the R.O.C.K. research group, University of Trento, and from the "Fonds de la Recherche Fondamentale Collective" (research grant "Preference dynamics in adaptive networks", $\mathrm{n}^{\circ}$ 2.4614.12). The funding sources had no role in the design of the present experiment, in the collection, analysis, and interpretation of data, in the writing of the report, and in the decision to submit the paper for publication. The usual caveats apply.

\section{Conflicts of Interest}

The authors declare no conflict of interest. 


\section{References}

1. McKelvey, R.D.; Palfrey, T.R. Quantal response equilibrium for normal form games. Games Econ. Behav. 1995, 10, 6-38.

2. Eyster, E.; Rabin, M. Cursed equilibrium. Econometrica 2005, 73, 1623-1672.

3. Stahl, D.O.; Wilson, P.W. Experimental evidence on players' models of other players. J. Econ. Behav. Organ. 1994, 25, 309-327.

4. Nagel, R. Unraveling in guessing games: An experimental study. Am. Econ. Rev. 1995, 85, 1313-1326.

5. Stahl, D.O.; Wilson, P.W. On players' models of other players: Theory and experimental evidence. Games Econ. Behav. 1995, 10, 218-254.

6. Costa-Gomes, M.; Weizsäcker, G. Stated beliefs and play in normal-form. Games Rev. Econ. Stud. 2008, 75, 729-762.

7. Weizsäcker, G. Ignoring the rationality of others: Evidence from experimental normal-form games. Games Econ. Behav. 2003, 44, 145-171.

8. Devetag, G.; Warglien, M. Playing the wrong game: An experimental analysis of relational complexity and strategic misrepresentation. Games Econ. Behav. 2008, 62, 364-382.

9. Rydval, O.; Ortmann, A.; Ostatnicky, M. Three very simple games and what it takes to solve them. J. Econ. Behav. Organ. 2009, 72, 589-601.

10. Cox, J.C.; Smith, V.L.; Walker, J.M. Experimental development of sealed-bid auction theory; calibrating controls for risk aversion. Am. Econ. Rev. 1985, 75, 160-165.

11. Goeree, J.K.; Holt, C.A.; Palfrey, T.R. Quantal response equilibrium and overbidding in private-value auctions. J. Econ. Theory 2002, 104, 247-272.

12. Holt, C.A.; Laury, S.K. Risk aversion and incentive effects. Am. Econ. Rev. 2002, 92, 1644-1655.

13. Goeree, J.K.; Holt, C.A.; Palfrey, T.R. Risk averse behavior in generalized matching pennies games. Games Econ. Behav. 2003, 45, 97-113.

14. Schelling, T.C. The Strategy of Conflict; Oxford University Press: New York, NY, USA, 1963.

15. Sugden, R. Thinking as a team: Towards an explanation of nonselfish behavior. Soc. Philos. Policy 1993, 10, 69-89.

16. Mehta, J.; Starmer, C.; Sugden, R. The nature of salience: An experimental investigation of pure coordination games. Am. Econ. Rev. 1994, 84, 658-673.

17. Tversky, A. Features of similarity. Psychol. Rev. 1977, 84, 327-352.

18. Holland, J.H.; Holyoak, K.J.; Nisbett, R.E.; Thagard, P.R. Induction: Process of Inference, Learning, and Discovery; MIT Press: London, UK, 1986.

19. Rubinstein, A. Similarity and decision-making under risk (is there a utility theory resolution to the Allais Paradox?). J. Econ. Theory 1988, 46, 145-153.

20. Leland, J.W. Generalized similarity judgments: An alternative explanation for choice anomalies. J. Risk Uncertain. 1994, 9, 151-172.

21. Leland, J.W. Similarity judgments in choice under uncertainty: A reinterpretation of the predicitions of regret theory. Manag. Sci. 1998, 44, 659-672.

22. Gilboa, I.; Schmeidler, D. Case-based decision theory. Q. J. Econ. 1995, 110, 605-639.

23. LiCalzi, M. Fictitious play by cases. Games Econ. Behav. 1995, 11, 64-89. 
24. Jehiel, P. Analogy-based expectation equilibrium. J. Econ. Theory 2005, 123, 81-104.

25. Grimm, V.; Mengel, F. An experiment on learning in a multiple games environment. J. Econ. Theory 2012, 147, 2220-2259.

26. Knez, M.; Camerer, C. Increasing cooperation in Prisoner's dilemmas by establishing a precedent of efficiency in coordination games. Organ. Behav. Hum. Decis. Processes 2000, 82, 194-216.

27. Rankin, F.W.; van Huyck, J.B.; Battalio, R.C. Strategic similarity and emergent conventions: Evidence from similar stag hunt games. Games Econ. Behav. 2000, 32, 315-337.

28. Kahneman, D.; Tversky, A. Prospect theory: An analysis of decision under risk. Econometrica 1979, 47, 263-292.

29. Tversky, A.; Kahneman, D. The framing of decisions and the psychology of choice. Science 1981, 211, 453-458.

30. Goeree, J.K.; Holt, C.A. Ten little treasures of game theory and ten intuitive contradictions. Am. Econ. Rev. 2001, 91, 1402-1422.

31. Camerer, C.F. Behavioral Game Theory: Experiments in Strategic Interaction; Princeton University Press: Princeton, NJ, USA, 2003.

32. Crawford, V.P.; Gneezy, U.; Rottenstreich, Y. The power of focal points is limited: Even minute payoff asymmetry may yield large coordination failures. Am. Econ. Rev. 2008, 98, 1443-1458.

33. Sugden, R. A theory of focal points. Econ. J. 1995, 105, 533-550.

34. Bosch-Domènech, A.; Vriend, N.J. On the Role of Non-Equilibrium Focal Points as Coordination Device; Working Paper, No. 621; Queen Mary University: London, UK, 2008.

35. Warglien, M.; Devetag, G.; Legrenzi, P. I Modelli mentali dei Giochi: Focalizzazione e rappresentazioni selettive. Sist. Intell. 1999, XI, 85-111, (in Italian).

36. Fischbacher, U. Z-Tree: Zurich toolbox for ready-made economic experiments. Exp. Econ. 2007, 10, 171-178.

37. Costa-Gomes, M.; Crawford, V.P.; Broseta, B. Cognition and behavior in normal-form games: An experimental study. Econometrica 2001, 69, 1193-1235.

38. Burnham, K.P.; Anderson, D.R. Model Selection and Multimodel Inference; Springer: New York, NY, USA, 2002.

39. Bhatt, M.; Camerer, C.F. Self-referential thinking and equilibrium as states of mind in games: fMRI evidence. Games Econ. Behav. 2005, 52, 424-459.

40. Rubinstein, A. Instinctive and cognitive reasoning: A study of response times. Econ. J. 2007, 117, 1243-1259.

41. Piovesan, M.; Wengström, E. Fast or fair? A study of response times. Econ. Lett. 2009, 105, 193-196.

42. Kuo, W.; Sjöström, T.; Chen, Y.; Wang, Y.; Huang, C. Intuition and deliberation: Two systems for strategizing in the brain. Science 2009, 324, 519-522.

(C) 2013 by the authors; licensee MDPI, Basel, Switzerland. This article is an open access article distributed under the terms and conditions of the Creative Commons Attribution license (http://creativecommons.org/licenses/by/3.0/). 